The Journal of Rheumatology 2021;48:6

doi: $10.3899 /$ jrheum. 201636

First Release March 152021

\section{Dr. Triantafyllias, et al reply}

\section{To the Editor:}

We have read with great interest the letter of Verhoeven, $e t$ al, ${ }^{1}$ referring to our recent publication on the diagnostic value of optical spectral transmission (OST) in rheumatoid arthritis (RA). ${ }^{2}$ In our work we had described for the first time, to our knowledge, that OST values could be influenced not only by disease-associated factors (i.e., inflammatory activity) but also by patient-associated characteristics, such as sex, BMI, and age. ${ }^{2}$ Moreover, we showed that patients with RA had higher OST values than controls and that OST was associated with clinical, ultrasonographic, and laboratory disease activity markers. ${ }^{2}$

Subsequently, Verhoeven, et al also examined associations between patient-related characteristics and OST values and found, among others, higher OST values in male controls (and probably RA patients), as well as in study subjects with larger or more voluminous hands. ${ }^{1}$ We are pleased to note that these results are in line with our recent findings and constitute a further step toward a better understanding of this new and exciting technology. We intend to conduct further studies, also in cooperation with other centers performing research on OST, in order to examine thoroughly the real effects of these and further patient characteristics (i.e., BMI, age, osteoarthritis). In doing so, we aim to contribute to an improvement of the diagnostic value of OST. As stated in our manuscript, we believe that valid interpretation of OST results presupposes consideration of all possible confounding patient- and disease-associated factors. ${ }^{2}$

Finally, we agree with the potential role of OST in the follow-up examinations of patients with inflammatory arthritis. In fact, the first results, to our knowledge, on the ability of OST to detect changes of inflammatory activity caused by antiinflammatory therapy have been recently presented by our group. ${ }^{3}$ Completion of these examinations, which also had been partially postponed due to the pandemic, and communication of the final results are currently ongoing.
Konstantinos Triantafyllias ${ }^{1}[$, MD

Caroline Heller ${ }^{2}$

Michele de Blasi ${ }^{1}$, Dipl. Ing.

Peter Robert Galle ${ }^{3}$, MD, Professor

Andreas Schwarting 1,2 (D), MD, Professor

${ }^{1}$ ACURA Rheumatology Center Rhineland-Palatinate, Bad Kreuznach;

${ }^{2}$ Internal Medicine I, Department of Rheumatology and Clinical

Immunology, Johannes Gutenberg University Medical Center, Mainz;

${ }^{3}$ Internal Medicine I, Department of Gastroenterology, University Medical

Center of the Johannes Gutenberg University, Mainz, Germany.

The authors declare no financial support or other benefits from commercial sources for the work reported in the correspondence, or any other financial interests that any of the authors may have, which could create a potential conflict of interest or the appearance of a conflict of interest with regard to the work.

Address correspondence to Dr. K. Triantafyllias, ACURA Rheumatology Center, Kaiser-Wilhelm-Str. 9-11, 55543, Bad Kreuznach, Germany.

Email: ktriantafyllias@gmail.com.

\section{REFERENCES}

1. Verhoeven MM, Westgeest AA, Jacobs JW. A sex difference in HandScan scores in rheumatoid arthritis patients and controls? An ongoing analysis of the sex difference and other potential confounders. J Rheumatol 2021;48:950-1.

2. Triantafyllias K, Heller C, de Blasi M, Galle P, Schwarting A. Diagnostic value of optical spectral transmission in rheumatoid arthritis: associations with clinical characteristics and comparison with joint ultrasonography. J Rheumatol 2020;47:1314-22.

3. Triantafyllias K, Heller C, de Blasi M, Muthuraman M, Schwarting A. Optical spectral transmission to assess therapy response in patients with arthritis: a comparative study with clinical, laboratory and ultrasonographic activity markers [abstract]. Ann Rheum Dis 2020;79 Suppl 1:1244. 